\title{
Importance of Family History of Colorectal Carcinoma In Situ Versus Invasive Colorectal Cancer: A Nationwide Cohort Study
}

Yu Tian, $\mathrm{PhD}^{1,2,3}$; Elham Kharazmi, MD, PhD ${ }^{1,4,5, *}$; Hermann Brenner, MD, $\mathrm{PhD}^{1,6,7}$; Xing Xu, $\mathrm{PhD}^{1,2}$; Kristina Sundquist, $\mathrm{MD}, \mathrm{PhD}^{4,8,9}$; Jan Sundquist, $\mathrm{MD}, \mathrm{PhD}^{4,8,9}$; and Mahdi Fallah, MD, $\mathrm{PhD}^{1,4,10, *}$

\begin{abstract}
Background: The aim of this study was to explore the risk of invasive colorectal cancer (CRC) in relatives of patients with colorectal carcinoma in situ (CCIS), which is lacking in the literature. Patients and Methods: We collected data from Swedish family-cancer datasets and calculated standardized incidence ratio (SIR) and cumulative risk of CRC in family histories of CCIS in first- and second-degree relatives. Family history was defined as a dynamic (time-dependent) variable allowing for changes during the follow-up period from 1958 to 2015. Of $12,829,251$ individuals with available genealogical data, 173,796 were diagnosed with CRC and 40,558 with CCIS. Results: The lifetime (0-79 years) cumulative risk of $\mathrm{CRC}$ in first-degree relatives of patients with CCIS was $6.5 \%$, which represents a 1.6 -fold $(95 \% \mathrm{Cl}, 1.5-1.7 ; n=752)$ increased risk. A similarly increased lifetime cumulative risk (6.7\%) was found among first-degree relatives of patients with CRC (SIR, 1.6; 95\% $\mathrm{Cl}, 1.6-1.7 ; n=6,965)$. An increased risk of $\mathrm{CRC}$ was also found in halfsiblings of patients with CCIS (SIR, 1.9; 95\% Cl, 1.1-3.0; $\mathrm{n}=18)$ and also in half-siblings of patients with CRC (SIR, 1.7; 95\% Cl, 1.3-2.1; $n=78$ ). Moreover, the increased risk of CRC was higher for younger age at diagnosis of CCIS in the affected first-degree relative and for younger age at diagnosis of CRC in the index person. Conclusions: Results of this study show that first-degree relatives and half-siblings of patients with CCIS have an increased risk of CRC, which is comparable in magnitude to the risk of those with a family history of invasive CRC. These findings extend available evidence on familial risk of CRC and may help to refine guidelines and recommendations for $\mathrm{CRC}$ screening.
\end{abstract}

J Natl Compr Canc Netw 2021;19(11):1252-1257 doi: 10.6004/jnccn.2021.7004

\footnotetext{
${ }^{1}$ Division of Preventive Oncology, German Cancer Research Center (DKFZ), and National Center for Tumor Diseases (NCT), and ${ }^{2}$ Medical Faculty Heidelberg, Heidelberg University, Heidelberg, Germany; ${ }^{3}$ School of Public Health, Capital Medical University, Beijing, China; ${ }^{4}$ Center for Primary Health Care Research, Lund University, Malmö, Sweden; ${ }^{5}$ Statistical Genetics Group, Institute of Medical Biometry and Informatics, Heidelberg University, Heidelberg, Germany; ${ }^{6}$ Division of Clinical Epidemiology and Aging Research, German Cancer Research Center (DKFZ), Heidelberg, Germany; ${ }^{7}$ German Cancer Consortium (DKTK), German Cancer Research Center (DKFZ), Heidelberg, Germany; ${ }^{8}$ Department of Family Medicine and Community Health, Department of Population Health Science and Policy, Icahn School of Medicine at Mount Sinai, New York, New York; ${ }^{9}$ Center for Community-based Healthcare Research and Education, Department of Functional Pathology, School of Medicine, Shimane University, Japan; and ${ }^{10}$ Department of Global Public Health and Primary Care, Faculty of Medicine, University of Bergen, Norway.

*These authors contributed equally to this study.
}

\section{Background}

Colorectal cancer (CRC) has become the third most common cancer and the second leading cause of cancer death globally. ${ }^{1}$ Colorectal carcinoma in situ (CCIS), which is defined as abnormal cells in the mucosa (innermost layer) of the colon/rectum wall, is the earliest stage of CRC. ${ }^{2}$ These abnormal cells may become cancerous and spread into nearby normal tissue, and are usually focal and situated on the superficial portion of the polyp., ${ }^{3,4}$

It is well-known that a family history of invasive CRC is associated with increased risk of invasive $\mathrm{CRC}^{5-7}$; however, there is less clarity regarding whether and to what extent family history of CCIS is associated with an increased risk of developing invasive CRC. Invasive CRC incidence in young adults below the age of screening (50 years) is increasing, and the discovery of any new risk factor that helps identify such young high-risk individuals would be of great importance. ${ }^{8,9}$ The highest familial risk of invasive CRC is seen in relatives of those who are diagnosed with early-onset invasive CRC. ${ }^{7,10}$

We aimed to explore and quantify the association between family history of CCIS and risk of invasive CRC in first- and second-degree relatives, using the world's largest record linkage of nationwide cancer registry and genealogical datasets from Sweden. We also intended to investigate whether the diagnosis of CCIS in a family should raise concern in other family members and prompt them to seek early medical advice about preventive measures, such as screening.

\section{Patients and Methods}

\section{Swedish Family-Cancer Datasets}

This study used the 2017 update of the Swedish FamilyCancer Datasets. In this update, information from the Multigeneration Register, national censuses, death notifi-

See JNCCN.org for supplemental online content. 
cations, and the Swedish Cancer Registry (started in 1958) were linked using unique personal national registration number. ${ }^{11}$ This record linkage study of multiple nationwide datasets included all individuals residing in Sweden and born since 1932 (offspring generation) and their parents (parental generation), totaling more than 12.8 million individuals with available genealogical information. During the follow-up of these individuals by the end of 2015, approximately 1.7 million primary invasive cancer diagnoses and approximately 0.5 million carcinoma in situ diagnoses were recorded. The completeness of the registry has been estimated to be $>96 \% .^{12}$

\section{Study Population}

All 12,829,251 individuals with available genealogical information who were living in Sweden from January 1, 1958, to December 31, 2015, were included in this study. The follow-up period started for each individual from the beginning of 1958, the birth year, or the immigration year, whichever was latest. The follow-up period ended when the individual was diagnosed with CRC, emigrated, died, or at the end of 2015, whichever was first. The datasets did not contain real national identification numbers. Instead, pseudonymized data with technical serial numbers were used. The study protocol was approved by the Lund Regional Ethics Committee (ref 2012/795).

\section{Family History Ascertainment}

Detailed information on family history of colorectal tumors for each subject during the follow-up period was obtained from our record linkage between the Multigeneration Register and the Swedish Cancer Registry. Therefore, we were able to acquire not only the final family history status of individuals at the end of study follow-up but also dynamic family history status reflecting changes during follow-up of the index person whenever a new relative was diagnosed with colorectal tumor. ${ }^{13}$ These 2 definitions of family history assessment are explained in supplemental eAppendix 1 (available with this article at JNCCN.org).

\section{Colorectal Tumors Ascertainment}

Data from the Swedish Cancer Registry (from 1958 to 2015) were used to identify CCIS and primary CRC. The diagnoses of CRC were classified according to a 4-digit diagnostic code of the International Classification of Diseases, Seventh Revision (ICD-7), and subsequent revisions in Sweden. The following ICD-7 codes were used to extract information on patients with CRC: 153, 153.0, 153.1, 153.2, 153.3, 153.4, 153.8, 153.9, 154, 154.0, and 154.8. Two kinds of codes for histopathologic type were available: ICD codes for oncology (ICD-O-2 or ICD-O-3 since 1993) and WHO/ HS/CANC/24.1 Histology Code (pathoanatomic diagnosis [PAD] since 1958), by which invasive and in situ colorectal tumors in the study were identified. Behavior code " 2 " as the fifth digit of the ICD-O-2/3 morphology code and value "4" as the third digit of PAD code were used to identify patients with CCIS. We regarded the pathologically verified CCIS as a synonym for adenoma with high-grade dysplasia. ${ }^{14}$ In this study, by the term (invasive) CRC we mean primary invasive CRC. Both types of colorectal tumors in family histories reported in our results are exclusive, unless otherwise specified; for example, the risk reported for family history of CCIS did not include those with both family history of CCIS and family history of CRC.

\section{Statistical Analyses}

Standardized incidence ratios (SIRs) were calculated to measure the risk of cancer in family members of patients with CCIS or CRC, as the ratio of observed to expected number of cases. The expected numbers were calculated from strata-specific person-years in those with a certain family history of colorectal tumor multiplied by strataspecific incidence rates in those without history of colorectal tumor in their first- and second-degree relatives. For stratification and adjustment, we used sex, 5-year age group, time period (1958-1964, 1965-1969, 1970-1974, 1975-1979, 1980-1984, 1985-1989, 1990-1994, 1995-1999, 2000-2004, 2005-2009, or 2010-2015), residential area (small cities in south Sweden, small cities in north Sweden, or large cities), and socioeconomic status (white-collar worker, blue-collar worker, professional, farmer, private, or other/unspecified). We also included information on hospital admission for obesity, alcoholism, and chronic obstructive pulmonary disease, a surrogate measure of heavy smoking in our adjustment models.

The age-specific cumulative risks were calculated for each family history group based on the following equations: age-specific annual incidence rate $=$ number of cases for each age divided by person-years for that age; cumulative incidence rate $=$ sum of each age-specific annual incidence rates from age 0 to certain ages (ie, sum of incidence rates from age 0-30 years was reported as cumulative incidence for age $30,0-40$ for age 40 , etc); cumulative risk $=1-\exp [-$ cumulative incidence rate]. Similarly, the lifetime cumulative risk (LCR; with lifetime assumed to be 0-79 years based on the average life expectancy in Europe in 2015 of 78 years) ${ }^{15}$ was calculated. The 95\% confidence intervals of SIR, LCR, and age-specific cumulative risk were calculated using Poisson approximation. All analyses were performed using SAS 9.4 (SAS Institute Inc).

\section{Results}

A total of 12,829,251 individuals with available genealogy information (with at least 1 known first-degree relative in our datasets) were included in this study $(6,527,022$ [50.9\%] were men), of which 173,796 were diagnosed with CRC and 
40,558 with CCIS. Follow-up was up to 58 years (median, 35 years). Only 1,738 (4.3\%) of patients with CCIS werelater diagnosed with CRC after an average interval of 6.3 years (median, 4 years). In total, at the time of their diagnosis, 164,242 patients with CRC had no family history of CCIS or CRC (94.5\%), 861 had a family history of only CCIS $(0.5 \%)$, and 8,458 had a family history of only invasive CRC (4.9\%).

Individuals with a CCIS diagnosis in a first-degree relative (and no such diagnosis in a second-degree relative) had a 1.6-fold (95\% CI, 1.5-1.7) increased risk of invasive CRC, and individuals with CCIS diagnosed in a second-degree relative (and no such diagnosis in a first-degree relative) had a 1.2-fold (95\% CI, 1.0-1.5) increased risk (Table 1). These risks were similar to those in individuals with corresponding family history of CRC (one first-degree relative: SIR, 1.6; 95\% CI, 1.6-1.7; one second-degree relative: SIR, 1.2; 95\% CI, 1.1-1.3). Even the LCRs of these 2 categories of family history of CCIS were rather similar to risks in individuals with corresponding family history of invasive CRC (one first-degree relative: $6.5 \%$ CCIS vs $6.7 \%$ invasive CRC; one seconddegree relative: $4.6 \%$ CCIS vs $5.1 \%$ invasive CRC), and so were cumulative risks by age of both groups, with similar curves with overlapping confidence intervals (Figure 1).

After further stratification by detailed type of relationship, the similarity of invasive CRC risk between family history of CCIS and family history of invasive CRC still existed in each stratum. For example, individuals whose father was diagnosed with CCIS had similar invasive CRC risk (SIR, 1.6; LCR, 6.8\%) to those whose father had invasive CRC (SIR, 1.6; LCR, 6.6\%) (Table 1).

Results of further stratification by type of relationship of affected family members showed that the invasive CRC risk in those with one half-sibling affected with CCIS was highly increased (SIR, 1.9; 95\% CI, 1.1-3.0), similar to the risk in those with one sibling affected with CCIS (SIR, 1.8; 95\% CI, 1.6-2.1) (Table 1). This similarity was also found in those with a history of invasive CRC in a halfsibling (SIR, 1.7; 95\% CI, 1.3-2.1) or a sibling (SIR, 1.7; 95\% CI, 1.6-1.8). However, we did not find any significant increase in risk of invasive CRC in those with one second-degree relative affected with CCIS other than a half-sibling (without any affected first-degree relatives), such as a grandparent (SIR, 1.2; 95\% CI, 0.9-1.7; data not shown), or an uncle/aunt (SIR, 1.0; 95\% CI, 0.6-1.5; data not shown).

After further stratification by sex, age at diagnosis of invasive CRC in the index person, and age at diagnosis of CCIS or invasive CRC in the affected first-degree relative, the similarity of increased risk of invasive CRC between those with family history of CCIS and those with family

Table 1. Risk of Invasive CRC by Family History of In Situ or Invasive Colorectal Tumor

\begin{tabular}{|c|c|c|c|c|c|c|c|c|}
\hline \multirow[b]{4}{*}{ Family History ${ }^{a}$} & \multicolumn{4}{|c|}{ Relative Risk } & \multicolumn{4}{|c|}{ Absolute Risk } \\
\hline & \multicolumn{4}{|c|}{ Tumor Type in Affected Relative } & \multicolumn{4}{|c|}{ Tumor Type in Affected Relative } \\
\hline & \multicolumn{2}{|c|}{ In Situ } & \multicolumn{2}{|c|}{ Invasive } & \multicolumn{2}{|c|}{ In Situ } & \multicolumn{2}{|c|}{ Invasive } \\
\hline & $\mathbf{n}$ & $\operatorname{SIR}^{\mathrm{b}}(95 \% \mathrm{CI})$ & $\mathbf{n}$ & $\operatorname{SIR}^{\mathrm{b}}(95 \% \mathrm{CI})$ & $\mathbf{n}$ & LCR $(95 \% \mathrm{Cl})$ & $\mathbf{n}$ & LCR $(95 \% \mathrm{Cl})$ \\
\hline Population & & & & & 137,819 & $4.2 \%(4.1-4.2)$ & 137,819 & $4.2 \%(4.1-4.2)$ \\
\hline $0 \mathrm{FDR}+0 \mathrm{SDR}$ & 164,242 & Ref & 164,242 & Ref & 128,925 & $4.1 \%(4.0-4.1)$ & 128,925 & $4.1 \%(4.0-4.1)$ \\
\hline $1 \mathrm{FDR}+0 \mathrm{SDR}$ & 752 & $1.6(1.5-1.7)$ & 6,965 & $1.6(1.6-1.7)$ & 658 & $6.5 \%(5.9-7.1)$ & 6,463 & $6.7 \%(6.5-6.9)$ \\
\hline Father + O SDR & 212 & $1.6(1.4-1.9)$ & 2,566 & $1.6(1.6-1.7)$ & 210 & $6.8 \%(5.3-8.3)$ & 2,539 & $6.6 \%(6.3-7.0)$ \\
\hline Mother + 0 SDR & 193 & $1.4(1.2-1.6)$ & 2,561 & $1.6(1.5-1.7)$ & 192 & $5.5 \%(4.3-6.6)$ & 2,526 & $6.7 \%(6.4-7.1)$ \\
\hline 1 sibling + 0 SDR & 223 & $1.8(1.6-2.1)$ & 1,127 & $1.7(1.6-1.8)$ & 212 & $11.0 \%(4.4-17.0)$ & 1,096 & $7.6 \%(7.1-8.1)$ \\
\hline 1 child +0 SDR & 124 & $1.6(1.4-2.0)$ & 711 & $1.5(1.4-1.6)$ & 44 & $6.6 \%(4.4-8.7)$ & 302 & $6.8 \%(5.7-7.9)$ \\
\hline$\geq 2$ FDRs +0 SDR & 11 & $3.2(1.6-5.8)$ & 478 & $2.7(2.5-3.0)$ & 10 & $11.0 \%(3.8-17.0)$ & 464 & $11.0 \%(10.0-13.0)$ \\
\hline 2 FDRs +0 SDR & 11 & $3.3(1.6-5.8)$ & 444 & $2.6(2.4-2.9)$ & 10 & $11.0 \%(3.8-17.0)$ & 431 & $11.0 \%(9.7-12.0)$ \\
\hline $1 \mathrm{FDR}+1 \mathrm{SDR}$ & 4 & $3.6(1.0-9.3)$ & 131 & $2.9(2.5-3.5)$ & 4 & $9.7 \%(0-21.0)$ & 128 & $9.3 \%(6.8-12.0)$ \\
\hline$\geq 1 \mathrm{SDR}+0 \mathrm{FDR}$ & 94 & $1.2(1.0-1.5)$ & 850 & $1.2(1.1-1.3)$ & 86 & $4.8 \%(3.1-6.5)$ & 822 & $5.2 \%(4.5-5.9)$ \\
\hline $1 \mathrm{SDR}+0 \mathrm{FDR}$ & 91 & $1.2(1.0-1.5)$ & 784 & $1.2(1.1-1.3)$ & 84 & $4.6 \%(2.9-6.3)$ & 756 & $5.1 \%(4.4-5.8)$ \\
\hline 1 half-sibling only ${ }^{c}$ & 18 & $1.9(1.1-3.0)$ & 78 & $1.7(1.3-2.1)$ & 17 & $7.7 \%(2.9-12.0)$ & 73 & $6.2 \%(4.6-7.8)$ \\
\hline $\begin{array}{l}1 \text { SDR only (excl. half- } \\
\text { sibling) }\end{array}$ & 73 & $1.1(0.8-1.4)$ & 706 & $1.2(1.1-1.3)$ & 67 & $3.6 \%(1.9-5.3)$ & 683 & $4.7 \%(4.0-5.5)$ \\
\hline
\end{tabular}

Abbreviations: CRC, colorectal cancer; FDR, first-degree relative; LCR, lifetime (0-79 years) cumulative risk; SDR, second-degree relative; SIR, standardized incidence ratio.

${ }^{a}$ Family history categories with a small number of cases $(<5)$ are not presented.

${ }^{\mathrm{b}}$ Adjusted for age, sex, calendar periods, residential areas, and socioeconomic status.

'Example: Those with only 1 half-sibling affected with colorectal carcinoma in situ had 1.9-fold increased risk of invasive CRC, and 7.7\% LCR, whereas those with only 1 half-sibling affected with invasive CRC had 1.7 -fold increased risk of invasive CRC, and $6.2 \%$ LCR. 


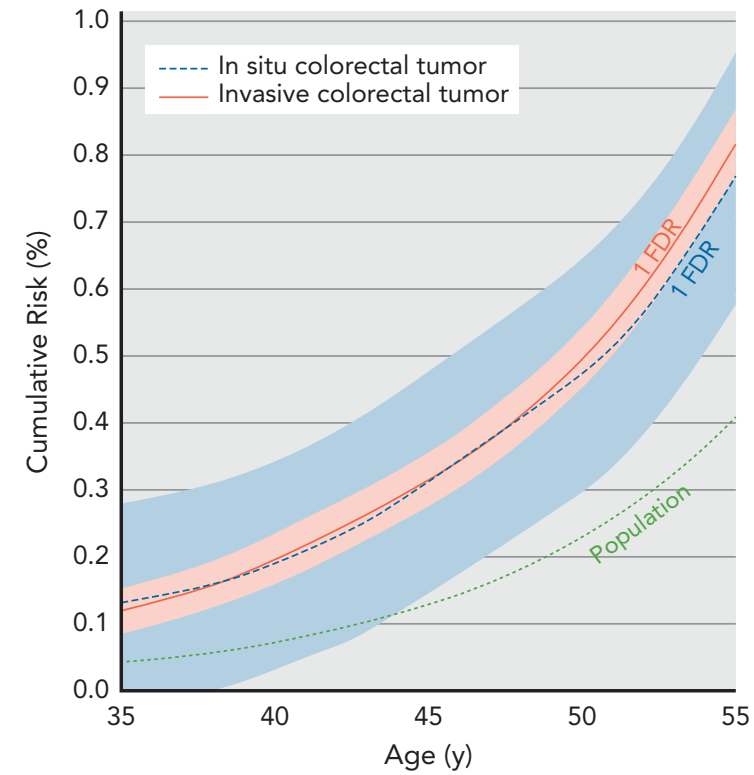

Figure 1. Cumulative risk of invasive colorectal cancer by age in those with an affected FDR with in situ or invasive colorectal tumor. Orange and blue shaded areas represent $95 \%$ confidence intervals for family history of invasive and in situ colorectal tumor, respectively. Abbreviation: FDR, first-degree relative.

history of invasive CRC was consistently observed in each stratum (Table 2). The highest relative risk was observed for invasive CRC diagnosed before age 60 years when the first-degree relative was also diagnosed with CCIS before age 60 years (SIR, 2.8; 95\% CI, 2.1-3.7). After exclusion of likely cases of hereditary nonpolyposis CRC according to the Amsterdam II criteria, the CRC risk remained unchanged (supplemental eAppendix 1).

\section{Discussion}

Using the world's largest nationwide datasets of its kind, we found that family history of CCIS in a first-degree relative or in a half-sibling is associated with an increased risk of invasive CRC. The magnitude of the risk increase is similar to that of having a family history of invasive CRC in corresponding family relationships. Interestingly, the risk of invasive CRC further significantly increased when one more first-degree relative (even one second-degree relative) was affected with CCIS. Furthermore, risk of earlyonset invasive CRC was higher when first-degree relatives were diagnosed with CCIS at earlier ages.

Many studies have suggested the increased familial CRC risk in first-degree relatives (to the best of our knowledge excluding CCIS from their familial risk estimates), ${ }^{5,16}$ but to date there is limited research on risk of invasive CRC in those with a family history of CCIS. Only one study, published in 1998, has examined the association of having a family history of colon or rectum carcinoma in situ and risk of invasive CRC in parents and offspring, and found an increase in relative risk of invasive CRC. ${ }^{17}$ Limitations of that study were limited inclusion of information on relatives (siblings were not included), the fact that the risks could not be evaluated in detail because of a small number of cases, and the static definition of family history; that is, not taking into account the changes in family history status of individuals during follow-up. Nevertheless, our current results were consistent with these results from the preCRC screening era in Sweden, supporting our hypothesis that family history of CCIS is as important as family history of invasive CRC for risk of invasive CRC. In our study, we found almost identical risk of invasive CRC associated with family history of CCIS and with family history of invasive CRC in different combinations of family history, type of relationship, sex, age at diagnosis of index person, and age at diagnosis of the affected first-degree relative. These subgroup analyses could be considered as internal validation of our findings, and the consistency of results across subgroups underlines the robustness of the findings for potential use in clinical practice and consulting of family members of patients with either CCIS or invasive CRC.

A potential association between increased risk of invasive CRC and family history of advanced adenoma (adenoma $\geq 1 \mathrm{~cm}$ or high-grade dysplasia or tubulovillous/villous histology $)^{18}$ or advanced colorectal polyps (advanced adenoma or sessile serrated polyp $\geq 1 \mathrm{~cm}$ or sessile serrated polyp with cytologic dysplasia, or traditional serrated adenoma of any size) has been reported. The National Colorectal Cancer Roundtable has accordingly created sample letters to help endoscopists and clinicians identify patients with advanced colorectal polyps, understand risk factors, and even be mindful of the increased risk to patients and their family members. ${ }^{19}$ Similar to individuals with family history of invasive CRC, family members of patients with advanced polyps are also critical screening targets for the prevention of early-onset CRC. In some existing screening guidelines, individuals who have first-degree relatives with advanced colorectal polyps are recommended to start screening at younger ages in the same way as those who have first-degree relatives with invasive CRC. ${ }^{20-23}$ Our nationwide cohort study, using highquality register-based data with long-term follow-up, and pathologically verified CCIS and invasive CRC, responded to the call from experts to "take the bull by the horns" with regard to methodology, ${ }^{24}$ which could overcome methodologic shortcomings of previous studies ${ }^{21}$ and provide novel findings of great importance. Studies on precursor lesions may increase the understanding of cancer etiology and cancer progression and can provide rationales for its molecular basis and genetic links, given that the progression of genetic alteration in invasive CRC has been extensively studied. ${ }^{25,26}$ 
Table 2. Relative Risk of Invasive CRC by Sex, Age at Diagnosis, and Tumor Type

\begin{tabular}{|c|c|c|c|c|c|c|c|c|c|c|c|c|c|}
\hline \multirow{4}{*}{$\begin{array}{l}\text { Age at CRC } \\
\text { Diagnosis } \\
\text { of Index } \\
\text { Person }\end{array}$} & \multirow{4}{*}{$\begin{array}{c}\text { Age at } \\
\text { Diagnosis } \\
\text { in One } \\
\text { Affected } \\
\text { FDR }\end{array}$} & \multicolumn{4}{|c|}{ All } & \multicolumn{4}{|c|}{ Men } & \multicolumn{4}{|c|}{ Women } \\
\hline & & \multicolumn{4}{|c|}{ Tumor Type in Affected Relative } & \multicolumn{4}{|c|}{ Tumor Type in Affected Relative } & \multicolumn{4}{|c|}{ Tumor Type in Affected Relative } \\
\hline & & \multicolumn{2}{|r|}{ In Situ } & \multicolumn{2}{|r|}{ Invasive } & \multirow[b]{2}{*}{$\mathbf{n}$} & \multirow{2}{*}{$\frac{\text { In Situ }}{\operatorname{SIR}^{\mathrm{b}}(95 \% \mathrm{CI})}$} & \multirow{2}{*}{\multicolumn{2}{|c|}{$\begin{array}{l}\text { Invasive } \\
\operatorname{SIR}^{\mathrm{b}}(95 \% \mathrm{CI})\end{array}$}} & \multirow[b]{2}{*}{$\mathbf{n}$} & \multirow{2}{*}{$\begin{array}{l}\text { In Situ } \\
\operatorname{SIR}^{\mathrm{b}}(95 \% \mathrm{CI})\end{array}$} & \multicolumn{2}{|r|}{ Invasive } \\
\hline & & $\mathbf{n}$ & $\operatorname{SIR}^{\mathrm{a}}(95 \% \mathrm{CI})$ & $\mathbf{n}$ & $\operatorname{SIR}^{\mathrm{a}}(95 \% \mathrm{CI})$ & & & & & & & $\mathbf{n}$ & $\operatorname{SIR}^{\mathrm{b}}(95 \% \mathrm{CI})$ \\
\hline \multirow[t]{3}{*}{ All } & All & 752 & $1.6(1.5-1.7)$ & 6,965 & $1.6(1.6-1.7)$ & 400 & $1.6(1.5-1.8)$ & 3,793 & $1.6(1.6-1.7)$ & 352 & $1.6(1.4-1.7)$ & 3,172 & $1.6(1.5-1.6)$ \\
\hline & $<60 y$ & 229 & $1.9(1.6-2.1)$ & 1,893 & $1.9(1.8-1.9)$ & 121 & $2.1(1.7-2.5)$ & 973 & $1.9(1.8-2.1)$ & 108 & $1.7(1.4-2.1)$ & 920 & $1.8(1.7-1.9)$ \\
\hline & $\geq 60 y$ & 523 & $1.5(1.4-1.6)$ & 5,072 & $1.5(1.5-1.6)$ & 279 & $1.5(1.3-1.7)$ & 2,820 & $1.6(1.5-1.6)$ & 244 & $1.5(1.3-1.7)$ & 2,252 & $1.5(1.4-1.6)$ \\
\hline \multirow[t]{3}{*}{$<60 y$} & All & 199 & $1.8(1.5-2.0)$ & 1,993 & $1.9(1.8-2.0)$ & 111 & $1.9(1.6-2.3)$ & 1,069 & $1.9(1.8-2.1)$ & 88 & $1.6(1.3-2.0)$ & 924 & $1.8(1.7-1.9)$ \\
\hline & $<60 y$ & 56 & $2.8(2.1-3.7)$ & 584 & $2.7(2.5-2.9)$ & 39 & $3.8(2.7-5.1)$ & 310 & $2.8(2.5-3.1)$ & 17 & $1.8(1.0-2.9)$ & 274 & $2.6(2.3-2.9)$ \\
\hline & $\geq 60 y$ & 143 & $1.5(1.3-1.8)$ & 1,409 & $1.7(1.6-1.8)$ & 72 & $1.5(1.2-1.9)$ & 759 & $1.7(1.6-1.9)$ & 71 & $1.6(1.2-2.0)$ & 650 & $1.6(1.5-1.8)$ \\
\hline \multirow[t]{3}{*}{$\geq 60 y$} & All & 553 & $1.6(1.4-1.7)$ & 4,972 & $1.5(1.5-1.6)$ & 289 & $1.6(1.4-1.7)$ & 2,724 & $1.5(1.5-1.6)$ & 264 & $1.5(1.4-1.7)$ & 2,248 & $1.5(1.4-1.6)$ \\
\hline & $<60 y$ & 173 & $1.7(1.5-2.0)$ & 1,309 & $1.6(1.6-1.7)$ & 82 & $1.7^{\mathrm{c}}(1.4-2.1)$ & 663 & $1.7(1.6-1.8)$ & 91 & $1.7(1.4-2.1)$ & 646 & $1.6(1.5-1.7)$ \\
\hline & $\geq 60 y$ & 380 & $1.5(1.3-1.7)$ & 3,663 & $1.5(1.4-1.5)$ & 207 & $1.5(1.3-1.7)$ & 2,061 & $1.5(1.4-1.6)$ & 173 & $1.5(1.3-1.7)$ & 1,602 & $1.5(1.4-1.5)$ \\
\hline
\end{tabular}

Abbreviations: CRC, colorectal cancer; FDR, first-degree relative; SIR, standardized incidence ratio.

${ }^{a}$ Adjusted for age, sex, calendar periods, residential areas, and socioeconomic status.

${ }^{\mathrm{b}}$ Adjusted for age, calendar periods, residential areas, and socioeconomic status.

'Example: men with 1 FDR affected with colorectal carcinoma in situ before age 60 years had 1.7-fold increased risk of invasive CRC at/after age 60 years.

Our study was based on data from latest available versions of several nationwide Swedish registers, which resulted in the world's largest study of its kind, assuring a high level of accuracy and completeness of the analyzed data. Using the data linkage between genealogy dataset and cancer registry to retrieve family history could mitigate several common biases, such as selection bias (exempted in a nationwide study), recall bias, and information bias (all required information was retrieved from record linkage, not self-reported during interviews); thus the study avoided biased estimates resulting from self-reports. ${ }^{27}$ Furthermore, this long-term cohort study allowed us to track CRC incidence over the lifetime with age-specific cumulative risk, which is an effect measure tangible for patients, their relatives, clinicians, researchers, and health policymakers. In addition, benefiting from large subgroup sample sizes, the study allowed precise quantification and comparison of the risk associated with the different categories of family history of colorectal tumors. However, some results in subgroup analyses (eg, for multiple firstdegree relatives affected with CCIS) were still based on relatively small sample sizes, and therefore some of risk estimates could be due to random variation. Nevertheless, the trend of risk by number of family members affected with CCIS was quite logical in these groups and was in line with that in multiple relatives affected with invasive CRC using a much larger sample size.

Although CCIS tumors in the Swedish Cancer Registry were $100 \%$ diagnosed based on microscopic examination of biopsied tissues, the registry data did not include information on type of polyps and whether diagnoses were based on polyps that were completely removed, partially removed, from visually abnormal sites in the colon or rectum that were subsequently biopsied, or from random biopsies of the colorectum. Some national screening guidelines recommend earlier screening if there had been a case of CRC in the family. In theory, such practices could lead to overestimation of the incidence of familial earlyonset CCIS after a patient with CRC was diagnosed in the family, but we used a dynamic approach to exclude such a bias. In fact, we considered the invasive CRC as the outcome of interest only when it occurred after the exposure to family history of CCIS, not the other way around. Moreover, there is no nationwide organized screening recommendation in Sweden for individuals with or without family history to lead to such a bias in the first place, even for invasive CRC. It should be noted that the definition and classification of advanced adenoma is not uniform in clinical practice, and challenges in subjectiveness and inaccurateness of assessment of size and histology of diagnosis of polyps are still inevitable. ${ }^{21,28}$ Some other limitations with no or trivial effect on our findings and further discussions on why dynamic family history was used can be found in supplemental eAppendix 1.

\section{Conclusions}

Our findings would supplement current screening guidelines by providing a strong recommendation, based on high-quality evidence, to consider family history of CCIS similarly to family history of invasive CRC in risk stratifications for earlier screening compared with the general population. We also recommend that future studies on familial risk of colorectal neoplasms should not only include family history of invasive CRC but also family history of CCIS. This may also lead to 
more precise estimates for attributable fraction and burden of familial colorectal neoplasms. Based on findings of this study, the evidence-based risk-adapted starting ages of CRC screening that we have recently provided for those with family history of invasive $\mathrm{CRC}^{7}$ should, in principle, also be applicable to those with family history of CCIS. Combining findings of these 2 robust studies would support screening guideline providers and clinicians with more detailed recommendations for those with family history of these conditions by number and degree of affected relatives as well as their age at diagnoses.

Submitted July 24, 2020; final revision received November 29, 2020; accepted for publication January 7, 2021. Published online September 13, 2021.
Author contributions: Study concept: Kharazmi, Fallah. Data curation: Kharazmi, Fallah. Formal analysis: Tian, Kharazmi, Fallah. Funding acquisition: Tian, Fallah. Investigation: Kharazmi. Methodology: Tian, Kharazmi, Fallah. Project administration: Kharazmi, Fallah. Resources: K. Sundquist, J. Sundquist. Software: Tian, Kharazmi, Xu. Supervision: Kharazmi, Fallah. Validation: Kharazmi, Fallah. Writing-original draft: Tian. Writing-review and editing: All authors.

Disclosures: The authors have disclosed that they have not received any financial consideration from any person or organization to support the preparation, analysis, results, or discussion of this article.

Funding: Xing Xu has received support from the China Scholarship Council.

Disclaimer: The funder had no role in the design and conduct of the study; collection, management, analysis, and interpretation of the data; preparation, review, or approval of the manuscript; and decision to submit the manuscript for publication.

Correspondence: Mahdi Fallah, MD, PhD, Division of Preventive Oncology, German Cancer Research Center (DKFZ) and National Center for Tumor Diseases (NCT), Im Neuenheimer Feld 581, 69120, Heidelberg, Germany. Email:m.fallah@dkfz.de

\section{References}

1. Sung H, Ferlay J, Siegel RL, et al. Global cancer statistics 2020: GLOBOCAN estimates of incidence and mortality worldwide for 36 cancers in 185 countries. CA Cancer J Clin 2021;71:209-249.

2. Amin MB, Edge S, Greene F, et al, eds. AJCC Cancer Staging Manual, 8th ed. New York, NY: Springer International Publishing; 2017.

3. National Cancer Institute. Stage 0 colorectal carcinoma in situ. Accessed May 29, 2020. Available at: https://www.cancer.gov/publications/ dictionaries/cancer-terms/def/stage-0-colorectal-carcinoma-in-situ

4. Fleming M, Ravula S, Tatishchev SF, et al. Colorectal carcinoma: pathologic aspects. J Gastrointest Oncol 2012;3:153-173.

5. Butterworth AS, Higgins JP, Pharoah P. Relative and absolute risk of colorectal cancer for individuals with a family history: a meta-analysis. Eur $J$ Cancer 2006;42:216-227.

6. Tian Y, Kharazmi E, Sundquist K, et al. Familial colorectal cancer risk in half siblings and siblings: nationwide cohort study. BMJ 2019;364:1803.

7. Tian $\mathrm{Y}$, Kharazmi $\mathrm{E}$, Brenner $\mathrm{H}$, et al. Calculating starting age for screening in relatives of patients with colorectal cancer based on data from large nationwide datasets. Gastroenterology 2020;159:159-168.

8. Siegel RL, Fedewa SA, Anderson WF, et al. Colorectal cancer incidence patterns in the United States, 1974-2013. J Natl Cancer Inst 2017;109: djw322.

9. Siegel RL, Torre LA, Soerjomataram I, et al. Global patterns and trends in colorectal cancer incidence in young adults. Gut 2019;68:2179-2185.

10. Kharazmi $E$, Fallah $M$, Sundquist $K$, et al. Familial risk of early and late onset cancer: nationwide prospective cohort study. BMJ 2012;345:e8076.

11. Hemminki K, Ji J, Brandt A, et al. The Swedish Family-Cancer Database 2009: prospects for histology-specific and immigrant studies. Int J Cancer 2010;126:2259-2267.

12. Barlow $L$, Westergren $K$, Holmberg $L$, et al. The completeness of the Swedish Cancer Register: a sample survey for year 1998. Acta Oncol 2009; 48:27-33.

13. Mukama T, Kharazmi E, Sundquist $K$, et al. Familial risk of breast cancer by dynamic, accumulative, and static definitions of family history. Cancer 2020;126:2837-2848

14. Bond JH. Polyp guideline: diagnosis, treatment, and surveillance for patients with nonfamilial colorectal polyps. The Practice Parameters Committee of the American College of Gastroenterology. Ann Intern Med 1993; 119:836-843.
15. World Health Organization. World health statistics 2016: monitoring health for the SDGs, sustainable development goals. Accessed May 29, 2020. Available at: http://www.who.int/iris/handle/10665/206498

16. Frank $\mathrm{C}$, Fallah $\mathrm{M}$, Sundquist J, et al. Population landscape of familial cancer. Sci Rep 2015;5:12891.

17. Hemminki K, Vaittinen P. Familial risks in in situ cancers from the FamilyCancer Database. Cancer Epidemiol Biomarkers Prev 1998;7:865-868.

18. Gupta S, Lieberman D, Anderson JC, et al. Recommendations for follow-up after colonoscopy and polypectomy: a consensus update by the US MultiSociety Task Force on Colorectal Cancer. Gastrointest Endosc 2020;91: 463-485.e5.

19. National Colorectal Cancer Roundtable. Advanced Colorectal Polyp Brief Accessed September 30, 2020. Available at: https://nccrt.org/resource/ advanced-colorectal-polyp-brief/

20. Molmenti CL, Kolb JM, Karlitz JJ. Advanced colorectal polyps on colonoscopy: a trigger for earlier screening of family members. Am J Gastroenterol 2020;115:311-314.

21. Kolb JM, Molmenti CL, Patel SG, et al. Increased risk of colorectal cancer tied to advanced colorectal polyps: an untapped opportunity to screen first-degree relatives and decrease cancer burden. Am J Gastroenterol 2020;115:980-988.

22. Rex DK, Boland CR, Dominitz JA, et al. colorectal cancer screening: recommendations for physicians and patients from the U.S. Multi-Society Task Force on Colorectal Cancer. Am J Gastroenterol 2017;112:1016-1030.

23. Leddin D, Lieberman DA, Tse F, et al. Clinical practice guideline on screening for colorectal cancer in individuals with a family history of nonhereditary colorectal cancer or adenoma: the Canadian Association of Gastroenterology Banff Consensus. Gastroenterology 2018;155:1325-1347.e3.

24. Kahi CJ, Lieberman D. Family history of colorectal adenomas: taking the methodological bull by the horns. Gastroenterology 2016;150:550-552.

25. Rubio CA, Delinassios JG. Invasive carcinomas may arise in colorectal adenomas with high-grade dysplasia and with carcinoma in situ. Int J Clin Exp Med 2010;3:41-47.

26. Armaghany $\mathrm{T}$, Wilson JD, Chu $\mathrm{Q}$, et al. Genetic alterations in colorectal cancer. Gastrointest Cancer Res 2012;5:19-27.

27. McGarragle KM, Hare $C$, Holter $S$, et al. Examining intrafamilial communication of colorectal cancer risk status to family members and kin responses to colonoscopy: a qualitative study. Hered Cancer Clin Pract 2019;17:16.

28. Terry MB, Neugut Al, Bostick RM, et al. Reliability in the classification of advanced colorectal adenomas. Cancer Epidemiol Biomarkers Prev 2002;11:660-663. 
Supplemental online content for:

\section{Importance of Family History of Colorectal Carcinoma In Situ Versus Invasive Colorectal Cancer: A Nationwide Cohort Study}

Yu Tian, PhD; Elham Kharazmi, MD, PhD; Hermann Brenner, MD, PhD; Xing Xu, PhD; Kristina Sundquist, MD, PhD; Jan Sundquist, MD, PhD; and Mahdi Fallah, MD, PhD

J Natl Compr Canc Netw 2021;19(11):1252-1257

eAppendix 1: Online Methods, Results, and Discussion eFigure 1: Schematic Diagram for the Definition of Static and Dynamic Family History eTable 1: Relative Risk of Invasive CRC by Sex and Family History of In Situ or Invasive Colorectal Tumor eTable 2: Relative Risk of Invasive CRC by Sex, Age at Diagnosis, and Tumor Type eTable 3: Relative Risk of CRC After Exclusion of Likely HNPCC Cases and HNPCC-Related Cancer in Families 


\section{eAppendix 1. Online Methods, Results, and Discussion}

\section{Online Methods \\ Family History}

In the static method, the final family history status of individuals during the whole study period is used independent of the family history timeline. This means that an individual is not considered to have a family history of colorectal carcinoma in situ (CCIS) if the family member was first diagnosed with CCIS and then the same family member was diagnosed colorectal cancer (CRC) after 10 years. In our analyses using the family history as a time-dependent (dynamic) variable, person-years of each individual were allocated to different risk categories based on family history status at each time period. For example, the time when no family member was affected until the time when the first member was diagnosed with tumor was allocated to category "having no family history of colorectal tumor." Similarly, the time from diagnosis of first affected family member until that of the second family member was classified as "having one affected family member," taking into account the sequence of occurrence of in situ or invasive colorectal tumor, number of affected first- and second-degree relatives, and age at diagnosis during the follow-up. When a CCIS occurred after CRC in the same relative, it was not included in this study. These 2 definitions of family history assessment are briefly presented by a schematic diagram in supplemental eFigure 1.

All types of relationships in family histories in our results are exclusive, meaning that, for example, the risk reported for one affected first-degree relative does not include those with both an affected first-degree relative and any other second-degree relatives. Siblings were defined as those who had common father and mother, whereas half-siblings (as second-degree relatives) were related through only one parent, either the father or mother.

The conventional cutoff point of age 60 years was chosen for diagnosis of CRC and CCIS in first-degree relatives similar to the age cutoff for risk stratification for those with family history in CRC screening guidelines (eg, by U.S. Multi-Society Task Force on Colorectal Cancer) and large population-based studies on familial risk of CRC. ${ }^{1-3}$

\section{Sensitivity Analyses}

To exclude the effect of hereditary CRC syndrome in familial risk calculations, we identified hereditary nonpolyposis CRC (HNPCC) cases in our datasets based on the Amsterdam II criteria. ${ }^{4}$ We even more strictly excluded all the families with any HNPCC-related cancer other than CRC (eg, endometrial, ovarian, small intestine, pancreatic, nervous system, liver, kidney, and urinary bladder).

\section{Online Results}

The lifetime cumulative risk of invasive CRC in those with one first-degree relative affected with invasive CRC and the other first-degree relative affected with CCIS was $10 \%(95 \%$ CI, $7.9 \%-13 \% ; \mathrm{n}=110$; standardized incidence ratio [SIR], 2.4; 95\% CI, 2.0-2.9; data not shown), rather similar to that in individuals with family history of 2 first-degree relatives affected with invasive CRC (11\%; SIR, 2.6; 95\% CI, 2.4-2.9; Table 1) with overlapping confidence intervals.

After further stratification by sex, invasive CRC risk in all types of family history of CCIS or invasive CRC remained the same in men and women (supplemental eTable 1).

The relative risk of invasive CRC was lower for older age at invasive CRC diagnosis in the index person and older age at diagnosis of CCIS in an affected first-degree relative (supplemental eTable 2). For example, the risk of invasive CRC diagnosis at older ages ( $\geq 70$ years) was 1.4 -fold increased when a first-degree relative was also diagnosed with CCIS at an advanced age (age at diagnosis $\geq 70$; SIR, 1.4; 95\% CI, 1.1-1.7).

After exclusion of likely HNPCC cases according to the Amsterdam II criteria, the CRC risk remained unchanged (supplemental eTable 3). Furthermore, after more strict exclusion of all families with any HNPCC-related cancer other than CRC, the similarity of invasive CRC risk between those with family history of CCIS and those with family history of invasive CRC did not substantially change. Moreover, our results did not vary after further adjustment for admission to hospital for obesity, chronic obstructive pulmonary disease (COPD), or alcoholism (data not shown).

\section{Online Discussion}

We observed a similar risk of invasive CRC in those whose full-siblings or half-siblings were diagnosed with CCIS compared with the risk in those with corresponding family history of invasive CRC. ${ }^{5}$ The similarity of risk in siblings (SIR, 1.8; 95\% CI, 1.6-2.1) and half-siblings (SIR, 1.9; 95\% CI, 1.1-3.0) with CCIS and dissimilarity to other seconddegree relatives may indicate that the family clustering of such cases of CRC may more likely be caused by shared 


\section{eAppendix 1. Online Methods, Results, and Discussion (cont.)}

childhood environment and lifestyle factors rather than genetic factors. Half-siblings, as second-degree relatives, share $25 \%$ of their genetic material. If the familial aggregation was mainly caused by genetic factors, the risk estimate in half-siblings would not be expected to be similar to that in siblings (who share $50 \%$ of their genetic material). Findings of our current study therefore further support conclusions from our previous study about the role of environmental and lifestyle factors in familial risk of invasive CRC in siblings and half-siblings. ${ }^{5}$ Large etiologic studies still are warranted to further disclose the underlying causes of the observed patterns of familial aggregation.

Except approximately $2 \%$ to $5 \%$ of CRC cases that can be accounted by well-established certain cancer-related genetic defects, such as nonpolyposis CRC or familial adenomatous polyposis, there are still $15 \%$ to $20 \%$ of CRC cases tending to run in the family, which is not completely understood, maybe due to inherited genetic predisposition, shared environmental factors, or a combination of these both. ${ }^{6}$ Therefore, we could not simply take family history for granted as surrogate for genetic risk in our study. We considered the time-varying nature of family history status of colorectal tumors. In reality, individuals with no family history of cancer at a certain age are a pool of those without family history in their entire life and those who will have relatives with cancer diagnosis in future.

Similarly, individuals at a certain age with family history of cancer in only one relative are a pool of those with such a family history in their entire life and those who will have other relatives with cancer diagnosis in future. The prediction of familial risk should reflect this fact to be able to be applicable to individuals at risk. That is why we used dynamic method of family history assessment. This makes our results more relevant for risk stratification in real-life settings compared with results based on the conventional static definition of family history in familial risk studies. In the conventional definition, only final family history status of individuals at the end of the study period is considered, even if the cancer in the family member was diagnosed after the cancer of the index person.

We had no detailed information on several risk and preventive factors of CRC that may contribute to clustering of CRCs in families, such as physical activity or dietary habits. However, our SIRs were alternatively adjusted for residential area, socioeconomic status, hospitalization for obesity, COPD, and alcohol drinking, which to some extent contained information on the abovementioned lifestyle-related factors. Moreover, it has been proposed that family members of patients with adenomatous polyps or CRC may be more likely to undergo screening and thus are more likely to receive a CRC diagnosis. ${ }^{7}$ Although we did not have information on screening, such as colonoscopy history, our recent sensitivity analysis on comparison of familial risk of invasive CRC by calendar period of diagnosis before and after the CRC screening era in Sweden showed no substantial difference in familial risk. $^{5}$

Our findings extend available evidence on familial risk of CRC and may help to refine guidelines and recommendation for CRC screening by providing high-quality evidence for inclusion of family history of CCIS as one of the criteria for risk-adapted screening at ages earlier than benchmark starting age of screening in the general population.

\section{References}

1. Rex DK, Boland CR, Dominitz JA, et al. Colorectal cancer screening: recommendations for physicians and patients from the U.S. Multi-Society Task Force on Colorectal Cancer. Am J Gastroenterol 2017;112:1016-1030.

2. Lowery JT, Ahnen DJ, Schroy PC III, et al. Understanding the contribution of family history to colorectal cancer risk and its clinical implications: a state-of-the-science review. Cancer 2016;122:2633-2645.

3. Samadder NJ, Curtin K, Tuohy TM, et al. Increased risk of colorectal neoplasia among family members of patients with colorectal cancer: a population-based study in Utah. Gastroenterology 2014;147:814-821.e5, quiz e15-16.

4. Vasen HF, Watson P, Mecklin JP, et al. New clinical criteria for hereditary nonpolyposis colorectal cancer (HNPCC, Lynch syndrome) proposed by the International Collaborative group on HNPCC. Gastroenterology 1999:116:1453-1456.

5. Tian Y, Kharazmi E, Sundquist K, et al. Familial colorectal cancer risk in half siblings and siblings: nationwide cohort study. BMJ 2019;364:1803.

6. Jasperson KW, Tuohy TM, Neklason DW, et al. Hereditary and familial colon cancer. Gastroenterology 2010;138:2044-2058.

7. Gupta S, Martinez E, Madlensky L. Colorectal cancer among first-degree relatives of individuals with adenomas: the risk is elevated, but now what? Cancer 2014;120: 4-6. 


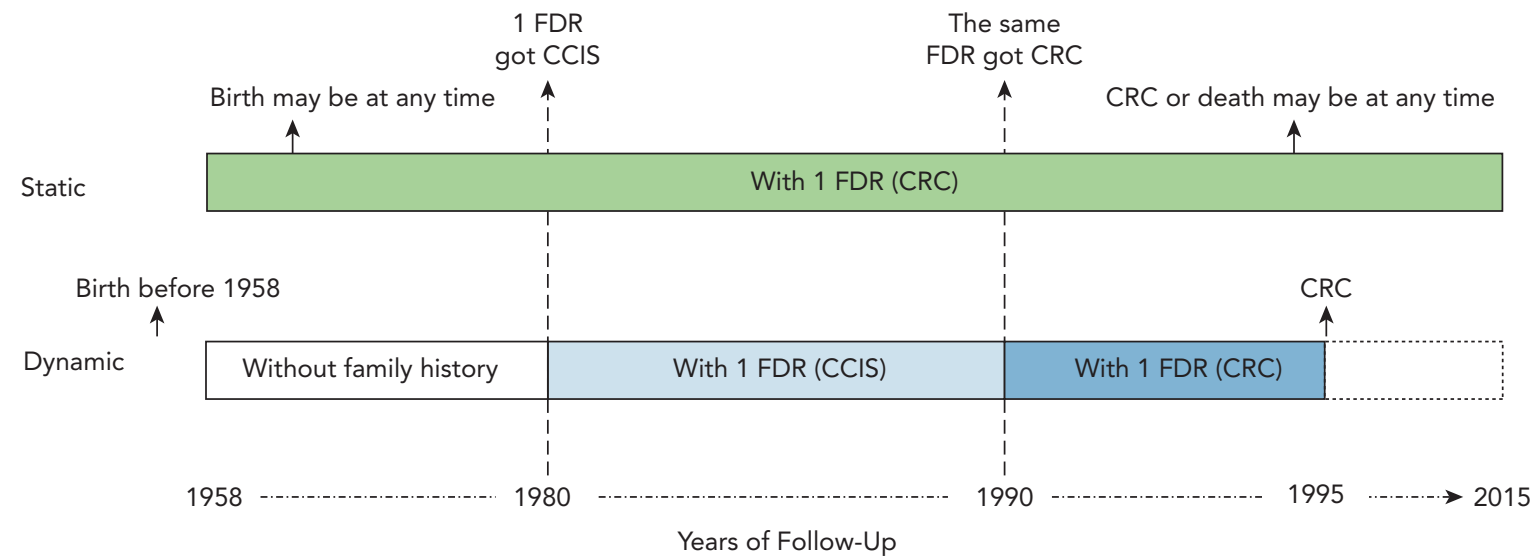

eFigure 1. Schematic diagram for the definition of static and dynamic family history. Example: In dynamic definition, the duration between 1980 (an FDR was diagnosed with CCIS) and 1990 (the same FDR was diagnosed with CRC) was counted as having the family history of CCIS in 1 FDR, whereas in the same person under static definition it was considered as with 1 FDR diagnosed with CRC for the entire study period (1958-2015). Abbreviations: CCIS, colorectal carcinoma in situ; CRC, colorectal cancer; FDR, first-degree relative. 
eTable 1. Relative Risk of Invasive CRC by Sex and Family History of In Situ or Invasive Colorectal Tumor

\begin{tabular}{|c|c|c|c|c|c|c|c|c|}
\hline \multirow{3}{*}{ Family History } & \multicolumn{4}{|c|}{ Men } & \multicolumn{4}{|c|}{ Women } \\
\hline & \multicolumn{4}{|c|}{ Tumor Type in Affected Relative } & \multicolumn{4}{|c|}{ Tumor Type in Affected Relative } \\
\hline & \multicolumn{2}{|c|}{ In Situ } & \multicolumn{2}{|c|}{ Invasive } & \multicolumn{2}{|r|}{ In Situ } & \multicolumn{2}{|c|}{ Invasive } \\
\hline $0 \mathrm{FDR}+0 \mathrm{SDR}$ & 86,323 & Ref & 86,323 & Ref & 77,919 & Ref & 77,919 & Ref \\
\hline $1 \mathrm{FDR}+0 \mathrm{SDR}$ & 400 & $1.6(1.5-1.8)$ & 3,793 & $1.6(1.6-1.7)$ & 352 & $1.6(1.4-1.7)$ & 3,172 & $1.6(1.5-1.6)$ \\
\hline Mother + O SDR & 112 & $1.5(1.2-1.8)$ & 1,467 & $1.7(1.6-1.8)$ & 81 & $1.3(1.0-1.6)$ & 1,094 & $1.5(1.4-1.6)$ \\
\hline 1 sibling +0 SDR & 136 & $2.0(1.7-2.4)$ & 630 & $1.8(1.6-1.9)$ & 87 & $1.6(1.3-2.0)$ & 497 & $1.7(1.6-1.9)$ \\
\hline 1 child + 0 SDR & 47 & $1.6(1.2-2.1)$ & 290 & $1.5(1.3-1.7)$ & 77 & $1.7(1.3-2.1)$ & 421 & $1.5(1.4-1.6)$ \\
\hline 2 FDRs +0 SDR & 6 & $3.1(1.1-6.7)$ & 260 & $2.9(2.5-3.2)$ & 5 & $3.5(1.1-8.1)$ & 184 & $2.4(2.1-2.8)$ \\
\hline $1 \mathrm{FDR}+1 \mathrm{SDR}$ & 1 & $1.6(0.0-9.2)$ & 74 & $3.2(2.5-4.0)$ & 3 & $6.0(1.2-18.0)$ & 57 & $2.7(2.0-3.5)$ \\
\hline
\end{tabular}

Abbreviations: CRC, colorectal cancer; FDR, first-degree relative; SDR, second-degree relative; SIR, standardized incidence ratio.

${ }^{a}$ Adjusted for age, calendar periods, residential areas, and socioeconomic status.

${ }^{b}$ Example: Men with only 1 half-sibling affected with colorectal carcinoma in situ had 2.2-fold increased risk of invasive CRC. 


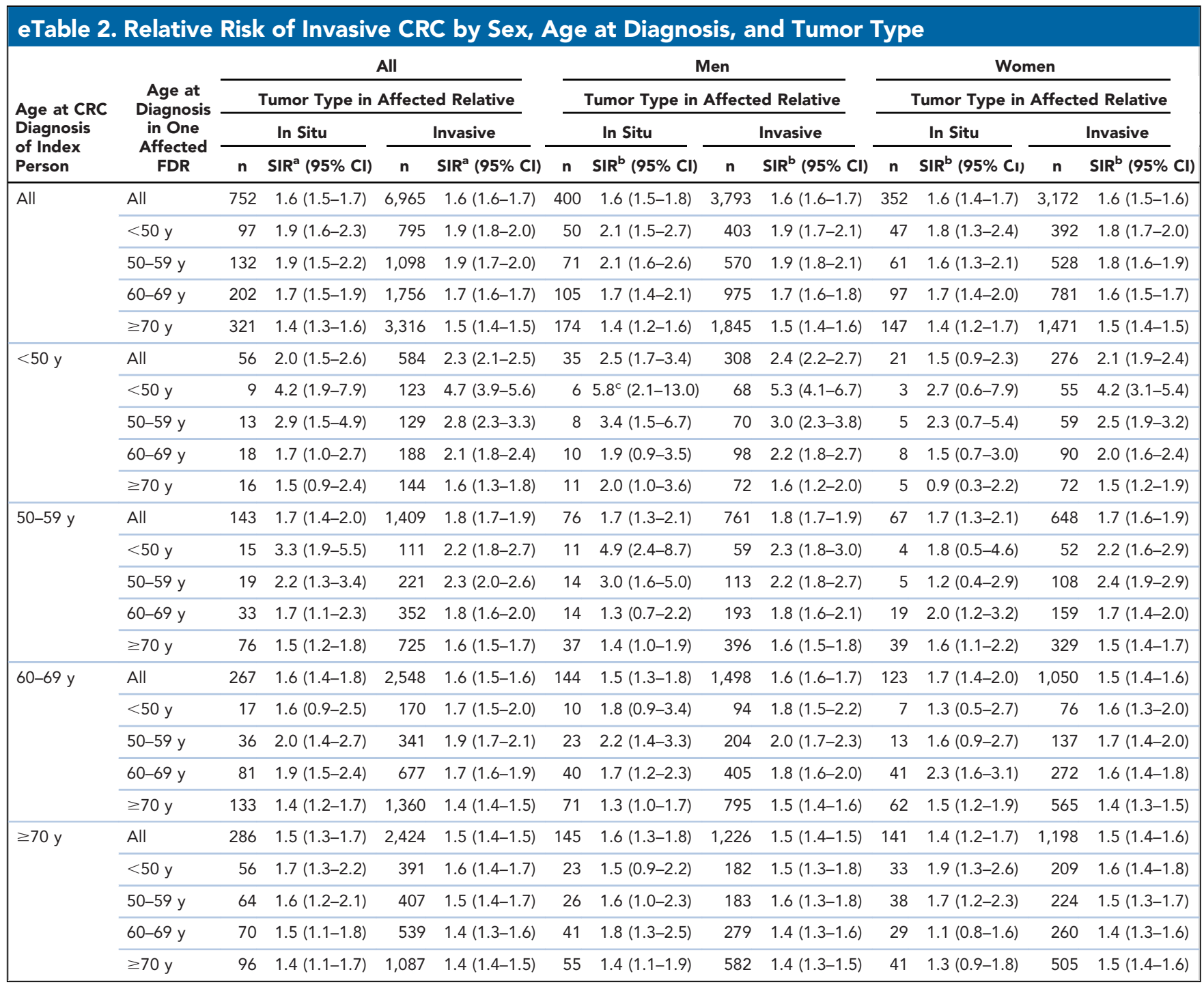

Abbreviations: CRC, colorectal cancer; FDR, first-degree relative; SIR, standardized incidence ratio.

${ }^{a}$ Adjusted for age, sex, calendar periods, residential areas, and socioeconomic status.

${ }^{b}$ Adjusted for age, calendar periods, residential areas, and socioeconomic status.

${ }^{\mathrm{C}}$ Example: men with 1 first-degree relative affected with colorectal carcinoma in situ before age 50 years had 5.8-fold increased risk of invasive CRC before age 50 years. 


\section{eTable 3. Relative Risk of CRC After Exclusion of Likely HNPCC Cases and HNPCC-Related Cancer} in Families

\begin{tabular}{|c|c|c|c|c|c|c|c|c|c|}
\hline \multirow{4}{*}{$\begin{array}{l}\text { Age at CRC } \\
\text { Diagnosis } \\
\text { of Index } \\
\text { Person }\end{array}$} & \multirow{4}{*}{$\begin{array}{l}\text { Age at } \\
\text { Diagnosis } \\
\text { in One } \\
\text { Affected } \\
\text { FDR }\end{array}$} & \multicolumn{4}{|c|}{ Exclusion of Likely HNPCC Cases } & \multicolumn{4}{|c|}{ Exclusion of Cases With HNPCC-Related Cancer } \\
\hline & & \multicolumn{4}{|c|}{ Tumor Type in Affected Relative } & \multicolumn{4}{|c|}{ Tumor Type in Affected Relative } \\
\hline & & \multicolumn{2}{|r|}{ In Situ } & \multicolumn{2}{|r|}{ Invasive } & \multicolumn{2}{|r|}{ In Situ } & \multicolumn{2}{|c|}{ Invasive } \\
\hline & & $\mathbf{n}$ & $\operatorname{SIR}^{\mathrm{a}}(95 \% \mathrm{Cl})$ & $\mathbf{n}$ & $\operatorname{SIR}^{\mathrm{a}}(95 \% \mathrm{CI})$ & $\mathbf{n}$ & $\operatorname{SIR}^{\mathrm{a}}(95 \% \mathrm{Cl})$ & $\mathbf{n}$ & $\operatorname{SIR}^{\mathrm{a}}(95 \% \mathrm{Cl})$ \\
\hline \multirow[t]{3}{*}{ All } & All & 752 & $1.6(1.5-1.7)$ & 6,949 & $1.6(1.6-1.7)$ & 497 & $1.5(1.4-1.7)$ & 4,849 & $1.6(1.5-1.6)$ \\
\hline & $<60 y$ & 229 & $1.9(1.6-2.1)$ & 1,883 & $1.9(1.8-1.9)$ & 154 & $1.8(1.5-2.1)$ & 1,281 & $1.7(1.6-1.8)$ \\
\hline & $\geq 60$ y & 523 & $1.5(1.4-1.6)$ & 5,066 & $1.5(1.5-1.6)$ & 343 & $1.4(1.3-1.6)$ & 3,568 & $1.5(1.5-1.5)$ \\
\hline \multirow[t]{3}{*}{$<60$ y } & All & 199 & $1.8(1.5-2.0)$ & 1,983 & $1.9(1.8-2.0)$ & 129 & $1.7(1.4-2.0)$ & 1,341 & $1.8(1.7-1.9)$ \\
\hline & $<60 y$ & 56 & $2.8(2.1-3.7)$ & 578 & $2.6(2.4-2.9)$ & 28 & $2.1(1.4-3.1)$ & 352 & $2.3(2.1-2.6)$ \\
\hline & $\geq 60$ & 143 & $1.5^{\mathrm{b}}(1.3-1.8)$ & 1,405 & $1.7(1.6-1.8)$ & 101 & $1.6(1.3-1.9)$ & 989 & $1.6(1.5-1.7)$ \\
\hline \multirow[t]{3}{*}{$\geq 60 \mathrm{y}$} & All & 553 & $1.6(1.4-1.7)$ & 4,966 & $1.5(1.5-1.6)$ & 368 & $1.5(1.3-1.6)$ & 3,508 & $1.5(1.4-1.5)$ \\
\hline & $<60 y$ & 173 & $1.7(1.5-2.0)$ & 1,305 & $1.6(1.6-1.7)$ & 126 & $1.7(1.4-2.0)$ & 929 & $1.6(1.5-1.7)$ \\
\hline & $\geq 60 y$ & 380 & $1.5(1.3-1.7)$ & 3,661 & $1.5(1.4-1.5)$ & 242 & $1.4(1.2-1.6)$ & 2,579 & $1.5(1.4-1.5)$ \\
\hline
\end{tabular}

Abbreviations: CRC, colorectal cancer; HNPCC, hereditary nonpolyposis colorectal cancer; FDR, first-degree relative; SIR, standardized incidence ratio.

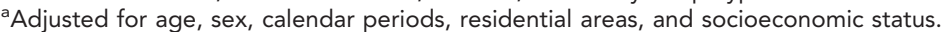

${ }^{b}$ Example: Men with 1 FDR affected with colorectal carcinoma in situ before age 60 years had 1.5 -fold increased risk of late-onset invasive CRC diagnosed after age 60 years. 